\title{
Consideraciones sobre la educación en prisión: un análisis de la realidad española a partir de la lectura de Stateville
}

\author{
Úrsula Ruiz Cabello \\ Universidad Pompeu Fabra \\ ursula.ruiz@upf.edu \\ José M. López-Riba \\ Universidad Pompeu Fabra \\ josemaria.lopez@upf.edu
}

Recepción: 31-10-2018

Aceptación: 08-03-2019

Publicación: 29-04-2019

\section{Resumen}

Este texto pertenece a una mesa redonda que ofrece un debate con James B. Jacobs, autor del clásico de la sociología de las prisiones Stateville: The penitentiary in mass society (1977), sobre la vigencia de su obra cuarenta años después. Este comentario tiene por objeto reflexionar en torno a las implicaciones de la educación en prisión a partir de la lectura de Stateville y del seminario que se impartió en la Universidad Pompeu Fabra para actualizar el debate sobre la monografía. En primer lugar, se expondrá el análisis de Jacobs acerca de la educación en prisión, subrayando la problemática de gestión que esta puede suponer. En segundo lugar, se apuntará la situación de la educación en las prisiones españolas. Para ello se señalarán los puntos en común con Stateville, se demostrarán los posibles beneficios que comporta la educación para los internos y, finalmente, se advertirá de las particularidades y precauciones que se deben considerar para poder garantizar el derecho a la educación a la población reclusa.

Palabras clave: prisión; educación; formación; gestión

Abstract. Remarks about education in prison: an analysis of the Spanish reality based on the reading of Stateville

This text forms part of a round table discussion with James B. Jacobs, author of the classic study on the sociology of prisons Stateville: The Penitentiary in Mass Society, and examines the validity of his work 40 years later. Specifically, the purpose of this commentary is to reflect on formal education in prison following the reading of Stateville and the seminar given at the University Pompeu Fabra aimed at revisiting the debate on Jacobs' work. The authors' analysis of education in prison is first presented, highlighting the management problems involved. The state of education in Spanish prisons, the points it shares with Stateville and the possible benefits of education for inmates are then discussed. Finally, particular aspects and precautions that must be considered in order to guarantee inmates' right to education are explored.

Keywords: prison; education; training; management 


\title{
Sumario
}

\author{
1. Introducción \\ 2. Breves notas sobre la educación \\ 3. La dificultad de conjugar la educación \\ y el orden en prisión \\ en las prisiones españolas \\ 4. Conclusiones \\ Referencias bibliográficas
}

\section{Introducción}

La prisión de Stateville, desde su origen, ofreció oportunidades de educación formal a sus internos: se impartían cursos formativos por parte de profesores universitarios y también, ocasionalmente, algunos internos tenían la oportunidad de salir de prisión para realizar el curso en la propia universidad (Jacobs, 1977). En un inicio, esta colaboración entre el mundo académico y la prisión era puntual. No obstante, a partir de los años sesenta, la cooperación entre la prisión y la universidad se fue estrechando.

A causa de la tesis principal de la monografía, Jacobs (1977) examina la educación formal en prisión, principalmente, como una cuestión de administración y gestión (management) del centro penitenciario. De acuerdo con el autor (1977), existen dos razones por las que la educación puede ser considerada un entorpecimiento de la gestión del centro: por un lado, la entrada de formadores puede suponer un disturbio del orden de la cárcel y complicar el funcionamiento de la prisión en términos de horarios, del personal requerido para el control y la vigilancia o del uso de los espacios. Por otro lado, existe cierta desconfianza hacia el contacto con el profesorado, ya que algunos funcionarios de prisión creen que los profesores pueden facilitar a los internos una mayor toma de conciencia de sus derechos y alentar sus reivindicaciones ${ }^{1}$.

A pesar de los problemas de gestión, para Jacobs la educación desempeña un papel importante en el presidio, ya que en el debate el profesor puso de manifiesto los impactos positivos que esta puede tener para los internos según la investigación criminológica (Jacobs, 2018).

Teniendo en cuenta que la educación supone, en ocasiones, una molestia para el control del orden en prisión, pero a su vez un claro beneficio para las personas internadas, planteamos al profesor Jacobs una pregunta acerca del impacto que puede tener la educación formal en la vida de las personas presas. En su respuesta, el profesor Jacobs (2018) resaltó el interés que suscita la línea de investigación que mide el impacto de los programas universitarios y formativos sobre los internos. Además, añade que el contacto con la academia también es provechoso en sí mismo para la propia institución carcelaria, puesto

1. Un ejemplo de tal desconfianza es la manifestada hacia los profesores provenientes de la Universidad del Norte de Illinois, que se sospechaba que acudían a dar clases porque tenían motivos ocultos para entrar en prisión, más allá de la educación (Jacobs, 1977: 57). 
que permite romper su aislamiento y la hace más transparente. En atención a la respuesta del sociólogo, el objeto de este artículo es indicar cómo el derecho penitenciario español ha hecho frente al dilema que presenta la educación en prisión, en tanto que es un derecho, pero cuyo disfrute puede provocar problemas en la gestión y en el orden de la institución penitenciaria. Para ello, se analizará en primer lugar cómo se articula la educación dentro del sistema tratamental; en segundo lugar, la adecuación del derecho penitenciario y la propia prisión para dar cumplimiento a tal derecho, y finalmente los obstáculos de gestión a los que se enfrenta la prisión para dar cabida a la educación.

\section{Breves notas sobre la educación en las prisiones españolas}

En España, la educación es un derecho fundamental recogido en el artículo 27 de la Constitución Española (CE). De acuerdo con Rodríguez (2013), en el ámbito penitenciario, dicho derecho se incardina en la orientación reeducadora y resocializadora del castigo (art. 25.2 CE), porque se considera que la educación es un mecanismo de tratamiento penitenciario que dota de las herramientas necesarias a los internos para su reinserción ${ }^{2}$ (Gil, 2010). A causa de su estrecha vinculación con la figura del tratamiento, el legislador ha configurado la educación como un «derecho-deber» (Rodríguez, 2013:42). Esto explica que para motivar la participación de los internos en los programas educativos se use un sistema de incentivos, en forma de beneficios y recompensas, previsto en el artículo 119 del Reglamento Penitenciario (RP).

La oferta formativa en prisión incluye desde la educación reglada obligatoria (educación primaria y educación secundaria) hasta la formación universitaria, que adquiere más presencia en el sistema penitenciario español a partir del año 2000 (Gutiérrez, Viedma y Callejo, 2010), pasando por la formación profesional y otro tipo de enseñanzas más específicas (de idiomas, de salud, para jóvenes, etc.) (Rodríguez, 2013).

Para hacer efectivo el derecho a la educación, la institución penitenciaria, del mismo modo que sucedía en Stateville, ofrece dos opciones: o bien se dota de las suficientes condiciones materiales para ofrecer cursos formativos dentro del centro, en forma de bibliotecas, aulas e incluso módulos universitarios ${ }^{3}$, o bien posibilita la movilidad a los internos para el estudio mediante la figura de la salida programada (art. $114 \mathrm{RP}$ ), la modalidad de vida en régimen abierto pleno (art. $83 \mathrm{RP}$ ) o restringido (82 RP) o el traslado a otro centro penitenciario para acudir a un establecimiento educativo (art. 121. RP).

2. Tal y como señala Blazich (2007: 54), la educación en prisión debe conjugar «prácticas y marcos normativos entre el sistema penitenciario y el sistema educativo con lógicas de funcionamiento diferentes: en el primero la del castigo y el disciplinamiento, fundante del derecho penal y las prisiones; y en el segundo la lógica del desarrollo integral de los sujetos, fundante de la educación".

3. Todo ello regulado en la Ley Orgánica General Penitenciaria (LOGP), artículos 55 a 58. Por ejemplo, en Madrid V - Soto del Real y Madrid VI - Aranjuez existe un módulo de la Universidad Nacional de Educación a Distancia (UNED). 
Sobre los beneficios de la educación en prisión, la literatura concluye que los programas educativos en el entorno penitenciario tienen efectos positivos tanto durante la estancia en la prisión como tras la excarcelación. Algunas investigaciones criminológicas destacan que, por un lado, en cuanto a los efectos durante la condena, la participación en programas educativos mejora la autopercepción de los internos (Baranger, Rousseau, Mastrorilli y Matesanz, 2018), reduce las infracciones disciplinarias dentro de la prisión (Gerber y Fritsch, 1995) y favorece el alejamiento del mundo delincuencial y de los aspectos negativos de la vida carcelaria (Caballero, 1985). Por otro lado, algunos beneficios que se manifiestan tras el cumplimiento de la condena son: la reducción de la reincidencia en términos generales (Lochner y Moretti, 2004; Wilson, Gallagher y MacKenzie, 2000); la promoción de una identidad que fomenta la desistencia del delito (Baranger et al., 2018; Runell, 2018); el aumento de la probabilidad de conseguir un trabajo y un salario más alto (Gerber y Fritsch, 1995; Lochner y Moretti, 2004); la reducción de la impaciencia y el aumento de la aversión al riesgo (Lochner y Moretti, 2004); así como la continuación de la formación tras la excarcelación (Gerber y Fritsch, 1995; Runell, 2018).

Los servicios educativos en prisión, eventualmente, deben adecuarse a las particularidades de la población penitenciaria derivadas de las características de las personas internas. En cuanto al nivel educativo de la población penitenciaria, destaca que su formación es normalmente más baja que el de la población general (Gallego, Cabrera, Ríos y Segovia, 2010). En la tabla 1 se pueden observar los resultados de algunas investigaciones sobre el nivel educativo de los internos que muestran que la gran mayoría solo han cursado las etapas obligatorias de la educación (primaria y secundaria) y no han alcanzado las etapas superiores.

Este hecho implica que tanto la configuración de los programas educativos como la oferta de los mismos deben tener en cuenta los diferentes niveles

Tabla 1. Nivel educativo de las personas internas en los centros penitenciarios españoles

\begin{tabular}{lcc}
\hline Nivel educativo & $\begin{array}{c}\text { Fuente: Gutiérrez et al. } \\
(\mathbf{2 0 1 0 )}\end{array}$ & $\begin{array}{c}\text { Fuente: Gallego et al. } \\
(\mathbf{2 0 1 0})\end{array}$ \\
\hline Analfabetismo $^{1}$ & $11,8 \%$ & $1-7,3 \%$ \\
Estudios primarios (incompletos) $^{\text {Estudios primarios (completos) }}$ & $31,1 \%$ & - \\
Educación secundaria (primera etapa) $^{\text {Educación secundaria (segunda etapa) }}$ & $28 \%$ & $45,2 \%$ \\
Estudios universitarios (primer ciclo) & $17,3 \%$ & - \\
Estudios universitarios (segundo ciclo) & $8,8 \%$ & $15,5 \%$ \\
Estudios universitarios (tercer ciclo) & $2 \%$ & $5,4 \%$ \\
\hline
\end{tabular}

1. Para Bedmar y Fresneda (2000) la situación de estar en la cárcel cumpliendo condena sin estar alfabetizado es como una "doble exclusión» social, aunque en su estudio revelan que los presos «no tienen consciencia de ser analfabetos, ni se sienten limitados por ello" (2000: 134). Sobre las causas de la falta de alfabetización, ver Bedmar y Fresneda (2000: 133 y ss). 
educativos de los internos, las especificidades de la población penitenciaria (Caballero, 1985; Gutiérrez et al., 2010; Rodríguez, 2013) y los intereses de la misma (Bedmar y Fresneda, 2000).

Otro factor que dificulta la adecuación de los programas es la heterogeneidad de la propia población, conformada por los diferentes rangos de edad o las situaciones de exclusión y marginalidad (Blazich, 2007). En cuanto a este punto, se debe prestar atención a grupos específicos de internos y a sus necesidades. Por ejemplo, las personas extranjeras suelen presentar necesidades idiomáticas específicas, previsión recogida en el artículo 118.2 RP, y, en el caso de las mujeres, se destaca la necesidad de adaptar los programas educativos a sus singularidades y evitar los elementos sexistas en los mismos (Cervelló, 2006; Igareda, 2006; Yagüe, 2007).

\section{La dificultad de conjugar la educación y el orden en prisión}

Entre las condiciones de la prisión que pueden entorpecer la efectiva aplicación del derecho a la educación ${ }^{4}$ destacan las relativas al tiempo, los espacios y la gestión del centro penitenciario y del personal ${ }^{5}$. Como advierte Viedma (2003: 97-98):

La realidad de la prisión impone un contexto de seguridad, control de la comunicación y estrictas normas de funcionamiento interno sobre cualquier consideración académica. Quien tiene la responsabilidad de la vigilancia es quien impone siempre las normas de la intervención. Es decir, como en otros contextos, la actuación se ha de adaptar al espacio y no al contrario.

De la marcada gestión del tiempo propia de la institución carcelaria, se desprenden algunos problemas horarios, puesto que a veces se producen solapamientos con otras actividades y la persona interna se encuentra en la posición de tener que elegir a cuál acudir (Batchelder y Pippert, 2002). Esto es importante en los casos en los que los internos con situaciones económicas precarias, que representan una proporción muy significativa dentro de las prisiones, deben elegir entre participar en un programa formativo o en las actividades laborales remuneradas (Batchelder y Pippert, 2002; Rodríguez, 2013). Sobre los espacios habilitados para el estudio, Caballero (1985) indica que, en ocasiones, no existe un espacio adecuado para estudiar o bien porque la previsión espacial de la prisión no cuenta con ello o porque, en la práctica, el espacio se acaba usando para otros fines. Finalmente, por lo que respecta al orden de la prisión, como se ha expuesto supra, Jacobs (1977) ya expresa las incomodidades que puede suponer la gestión de la entrada de personas

4. También hay que tener en cuenta que la diversidad de establecimientos penitenciarios y regímenes de vida lleva a situaciones muy variadas respecto a la oferta y realización de la educación formal (Caballero, 1985).

5. Además de situaciones imprevistas que se generan por las propias condiciones de la cárcel (Bedmar y Fresneda, 2000). 
externas en la prisión o la desconfianza hacia la función o las actividades que se desarrollan en la misma. También, a causa de la preocupación por el orden en la institución, se restringe la comunicación con el exterior, lo que lleva a limitar el acceso a internet en los ordenadores. Dicha prohibición limita la tarea educativa, sobre todo teniendo en cuenta el uso, cada vez más habitual, de las TIC en la formación. Este obstáculo también dificulta la opción de ofrecer educación a distancia, que sería la solución a algunos de los impedimentos que la gestión y la administración de la prisión provocan (Gutiérrez et al., 2010; Rodríguez, 2013). De hecho, en opinión de Viedma (2003), la educación a distancia es el modelo de educación (universitaria) más apropiado por las particularidades de la prisión. Por último, en cuanto a las limitaciones de personal, Gil (2010) destaca que en doce años no se convocaron oposiciones para la especialidad de pedagogía del cuerpo técnico de instituciones penitenciarias.

\section{Conclusiones}

Retomando el debate con el profesor James B. Jacobs, coincidimos en que la educación formal en prisión no es solo un asunto que deba tratarse desde el punto de vista de la administración y gestión de la cárcel, puesto que la educación, tal como ocurre fuera de la prisión, tiene un papel muy relevante en la vida de las personas internas y en el propio ambiente de la institución.

En España, la educación es un derecho fundamental que, además, en prisión cumple un cometido resocializador. Por ello, en la medida de lo posible, la ley procura ponderar la seguridad y la educación incentivando la participación del interno en un programa educativo, permitiendo salidas y traslados para la formación o fomentando la colaboración entre la prisión y la universidad. No obstante, las limitaciones siguen siendo obvias por la idiosincrasia de la propia institución: horarios, espacios, recursos materiales y personales, etc. Y, en ocasiones, la realidad dificulta lo que dispone la ley. Este hecho puede llevar a cuestionar si es posible educar en la cárcel. En nuestra opinión, es posible, pero pensando la educación en prisión con todas las particularidades que conlleva, y no trasladando el sistema educativo que se oferta en la vida en libertad, ya que en esta institución se enfrenta a retos concretos, como el tratamiento, la población, los medios materiales y los personales reales.

Por todos estos motivos, consideramos que las dificultades que impone la prisión en el desarrollo de programas formativos deben soslayarse y se debe apostar por una oferta educativa comprensiva que tenga en cuenta las necesidades de todos los colectivos y que esté a disposición de cualquier persona interesada en participar en estos programas. 


\section{Referencias bibliográficas}

BAtchelder, J. S. y Pippert, J. M. (2002). «Hard time or idle time: Factors affecting inmate choices between participation in prison work and education programs». The Prison Journal, 82(2), 269-80. <https://doi.org/10.1177/003288550208200206>

Baranger, J.; Rousseau, D.; Mastrorilli, M. E. y Matesanz, J. (2018). «Doing Time Wisely: The Social and Personal Benefits of Higher Education in Prison». The Prison Journal, 98 (4), 490-513. <https://doi.org/10.1177/0032885518776380>

Bedmar. M. y FreSnEdA, M. D. (2000). «Excluidos y recluidos. Educación en la prisión». Pedagogía Social: Revista Interuniversitaria, 6-7, 12-145.

BlazICH, G. S. (2007). "La educación en contextos de encierro». Revista Iberoamericana de Educación (44), 53-60.

Caballero, J. (1985). «El estudio en prisión como instrumento de tratamiento». En: Víctor Sancha Mata y Juan José Miguel Tobal (comp.) Tratamiento penitenciario: su práctica. Madrid: Ministerio de Justicia, 159-171.

Cervelló, V. (2006). «Las prisiones de mujeres desde una perspectiva de género». Revista General de Derecho Penal Iustel (5), 1-24.

Gallego, M.; Cabrera, P.; Ríos, J. y Segovia, J. L. (2010). Andar 1 km en línea recta. La cárcel del siglo XXI que vive el preso. Madrid: Universidad Pontificia de Comillas.

GERBER, J. y FrITSCH, E. J. (1995). «Adult academic and vocational correctional education programs: A review of recent research». Journal of Offender Rehabilitation, 22 (1-2), 119-42. <https://doi.org/10.1300/J076v22n01_08>

GIL, C. (2010). «La acción pedagógica en las prisiones: posibilidades y límites». Revista Española de Pedagogía, 68 (245), 49-64.

GuTiÉRREZ, J.; VIEDMA, A. y CALLEJO, J. (2010). «Estudios superiores en la educación penitenciaria española: un análisis empírico a partir de los actores». Revista de Educación, (353), 443-68.

IgAREDA, N. (2006). «Mujeres, integración y prisión». Boletín Criminológico (86), 1-4.

Jacobs, J. B. (1977). Stateville. The Penitentiary in Mass Society. Londres: The University of Chicago Press.

- (2018). «Author meets critics: Stateville Revisited». III Conferencia Internacional del Máster en Criminología y Ejecución Penal (UPF). Barcelona, 11 de mayo.

LOCHNER, L. y MORETTI, E. (2004). «The effect of education on crime: Evidence from prison inmates, arrests, and self-reports». American Economic Review, 94 (1), 155-89. <https://doi.org/10.1257/000282804322970751>

RodríGUEZ, C. (2013). «El derecho a la educación en el sistema penitenciario español». Revista General de Derecho Penal (20), 1-44.

RunelL, L. L. (2018). "Arrested Development: Pursuing a Higher Education in Carceral Contexts». The Prison Journal, 98 (4), 470-89. <https://doi.org/10.1177/0032885518776379>

Viedma, A. (2003). «La educación a distancia en prisión. Estudio de los alumnos de la UNED internos en centros penitenciarios». RIED: Revista Iberoamericana de Educación a Distancia, 6 (2), 97-120.

Wilson, D. B.; Gallagher, C. A. y Mackenzie, D. L. (2000). "A meta-analysis of corrections-based education, vocation, and work programs for adult offenders». Journal of Research in Crime and Delinquency, 37 (4), 347-68. <https://doi.org/10.1177/0022427800037004001> 
YAGÜE, C. (2007). «Mujeres en prisión. Intervención basada en sus características, necesidades y demandas». Revista Española de Investigación Criminológica, 4 (5), $1-24$.

\section{Textos legales}

Constitución Española, 1978. Publicado en el BOE núm. 311 de 29 de diciembre de 1978.

Ley orgánica $1 / 1979$, de 26 de septiembre, general penitenciaria. Publicado en el $B O E$ núm. 5 de octubre de 1979 .

Real decreto 190/1996, de 9 de febrero, por el que se aprueba el Reglamento Penitenciario. Publicado en el BOE de 15 de febrero de 1996. 